\title{
Association between celiac disease and idiopathic dilated cardiomyopathy: a case report
}

\author{
Elisa Romagnoli • Elena Boldrini • \\ Antonello Pietrangelo
}

Received: 13 May 2010/ Accepted: 13 July 2010/Published online: 25 August 2010

(C) SIMI 2010

\section{Case presentation}

Dr. Boldrini: P.G., male, 66 year old was evaluated in our ward on 27 April 2009 for the persistence at home of watery diarrhoea and abdominal pain over the previous 10 days.

For these symptoms, he had taken rifaximine and loperamide without benefit. He denied fever, blood or mucus in the stools. He had neither family history of inflammatory bowel disease, nor had travelled abroad over the prior months. In his medical history, in 1997 an idiopathic dilated cardiomyopathy was diagnosed when the patient was admitted to the hospital for stroke. Since then he had taken warfarin, along with digoxin, ramipril, furosemide, lansoprazole; he reported intolerance to beta blockers.

Upon admission, the patient was conscious and co-operative, blood pressure 100/60 $\mathrm{mmHg}$, heart rate 90 beats/ min., and normal $\mathrm{O}_{2}$ saturation. Clinical examination was substantially normal; except for the presence of bilateral leg edema. BMI was 24. We performed an ECG (Fig. 1) that showed normal sinus rhythm with left bundle branch block, and a short run of ventricular tachycardia; an X-ray of the abdomen was normal, whereas a chest radiography revealed "cardiomegaly" with initial apical flow distribution.

Biochemistry showed PTL $496,000 / \mathrm{mm}^{3}$, the prothrombin time exceeding the therapeutic range, severe protein

This case record was selected for the "Casi Clinici Gymnasium" session at the 2009 SIMI Annual Congress in Rome, and was reviewed by a Committee composed of Maria Domenica Cappellini, Nicola Montano and Luigi Pagliaro.

E. Romagnoli $(\varangle) \cdot$ E. Boldrini $\cdot$ A. Pietrangelo

Unit of Internal Medicine II, University Hospital of Modena,

Via del Pozzo 71, 41100 Modena, Italy

e-mail: elyrom80@libero.it deficiency with $1.85 \mathrm{~g} / \mathrm{dl}$ albumin, elevation of CRP (3.56 mg/dl), mild hypokalemia, severe iron deficiency (lowered serum iron and serum ferritin without transferrin elevation).

Mild rehydration and albumin supplementation were started during the initial days, then replaced by total parenteral nutrition.

\section{Diagnosis}

Dr. Boldrini, Dr. Romagnoli: Thyroid function was normal and stool culture negative; both tissue transglutaminase $(100 \mathrm{U} / \mu \mathrm{l}) \mathrm{Ig} \mathrm{A}$ endomysial antibodies were positive, with total serum $\operatorname{IgA}$ in the normal range.

In order to perform upper gastrointestinal (UGI) endoscopy, warfarin was discontinued and replaced by enoxaparin: UGI endoscopy showed appreciable reduction with scalloped configuration; histopathology examination documented partial villous atrophy with increased number of intra-epithelial lymphocytes (Lesion 3A, Marsh Classification).

Echocardiography showed severe dilatation and global hypokinesis of left ventricle (ejection fraction 25\%) with functional mitral insufficiency, severe left atrial enlargement, presence of ventricular dyssynchrony.

Twelve hours after the upper GI endoscopy the patient presented confusion and dysarthria: a head CT scan showed an acute ischemic lesion in the left cerebellar hemisphere whereas carotid Doppler scan was normal. The symptoms disappeared on the following day. The patient was discharged after 20 days with a diagnosis of "celiac disease with severe malnutrition, cerebellar ischemic stroke (probably cardioembolic) in patient with idiopathic dilated cardiomyopathy". 

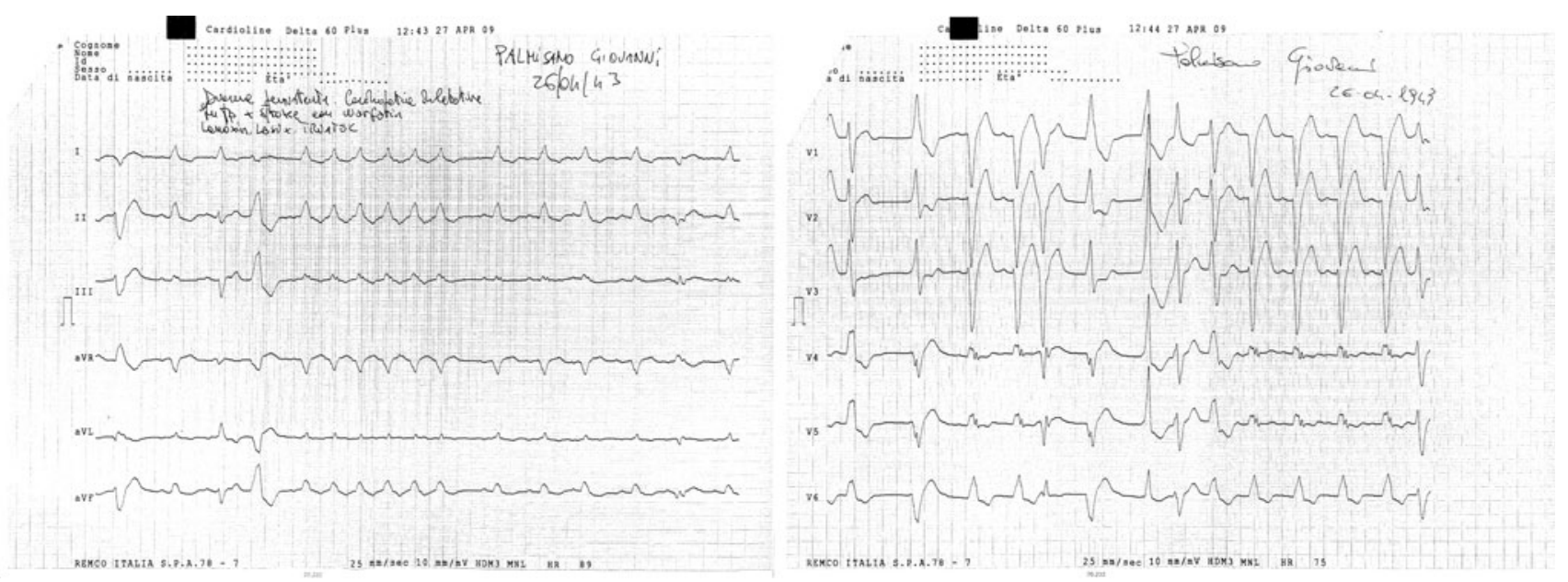

Fig. 1 ECG: normal sinus rhythm with left bundle branch block, and a short run of ventricular tachycardia

\section{Discussion}

Prof. Pietrangelo: The association of celiac disease and cardiomyopathy has been recently reported.

One study of 52 patients reports an increased prevalence of celiac disease $(5.7 \%)$ in patients with dilated cardiomyopathy [1]; in another study De Bem shows a prevalence of $2.7 \%$ for celiac disease in South Brazilian precardiac transplant patients with advanced cardiomyopathy [2]. An Italian study of 187 patients with myocarditis reveals that $4.4 \%$ of them are positive for antiendomysial antibodies with duodenal endoscopic and histological evidence of celiac disease [3]; Prati et al. [4] confirm that patients with end-stage heart failure are at increased risk for celiac disease as compared to the general population.

\section{Pathogenic mechanisms}

Prof Pietrangelo, Dr. Romagnoli: Several pathogenic mechanisms have been proposed for the development of cardiomyopathy in the course of celiac disease.

It is known that a reciprocal negative interaction between the heart and small intestine can occur when either organ is severely impaired. Intestinal absorption may be compromised in patients with congestive cardiac disease or idiopathic dilated cardiomyopathy, and celiac disease can affect the absorption of essential nutrients, leading to structural and functional abnormalities of cardiomyocytes that may exacerbate or precipitate an idiopathic dilated cardiomyopathy $[2,5]$.

Abnormalities of intestinal permeability in patients with celiac disease may lead to increased systemic absorption of various luminal antigens or infectious agents that may cause myocardial damage through immune-mediated mechanisms [6-8].
Finally, myocardial injury may be secondary to an immune response directed against an antigen present in both the myocardium and the small intestine [9].

The hypothesis of autoimmune disorders is strongly suggested by a number of data: the presence of serological detection of anti endomysial antibody and cardiac autoantibodies, the HLA profile, negative PCR studies for cardiotropic viruses, responsiveness to a gluten-free diet and immunosuppressive therapy [3].

Other authors propose that heart failure might result from other conditions associated with celiac disease as diabetes, thyroid disorders, vasculitis etc, in these cases anti endomysial antibody would merely "mark" a population with known risk for cardiac involvement [4].

As far as an inherited hypothesis is concerned, Not et al. find a higher prevalence of celiac disease in patients with sporadic or inherited dilated cardiomyopathy and a higher prevalence of IgA anti-tissue transglutaminase positive serology in relatives with echocardiographic abnormalities; these elements suggest that immune-mediated mechanisms are active in subsets of patients/families. However, gluten intolerance cannot be considered causative since celiac disease seems to be associated but does not co-segregate with dilated cardiomyopathy in familial cases [10].

\section{Role of diet}

Dr. Romagnoli, Dr. Boldrini: If the association between these two conditions were true, the gluten-free diet should have an important role. Frustaci et al. screened the serum of 187 consecutive patients with myocarditis: 13 were positive for IgA anti-tissue transglutaminase, and 9 (4.4\%) - 5 myocarditis associated with heart failure and 4 with ventricular dysrhythmias with Lown class III/IVa—exhibited duodenal endoscopic and histological evidence of $\mathrm{CD}$. The 
patients with myocarditis and heart failure received immunosuppression and a gluten-free diet, which elicited recovery of cardiac volumes and function. The patients with dysrhythmia, after being put on a gluten-free diet alone, showed improvement in the dysrhythmia (Lown class I). The authors conclude that in these patients immunosuppression and a gluten-free diet can be effective therapeutic options [3].

An Italian study investigated patients presenting idiopathic dilated cardiomyopathy associated with celiac disease: they show a greater decrease in serum total carnitine levels than patients presenting isolated form of idiopathic dilated cardiomyopathy; the gluten-free diet in these patients led to a progressive increase in serum levels of this micronutrient (an important cofactor for the metabolism of fatty acids that could play an important role in the improvement of heart performance) [11].

\section{True or false association?}

Dr. Romagnoli: Other authors do not find an association between celiac disease and subsequent myocarditis, cardiomyopathy or pericarditis in population studies [12]. Vizzardi et al. [13] indicate a similar prevalence of coeliac disease in patients with dilated cardiomyopathy in comparison to the Italian general population (1\%): for the authors the presence of confounding factor associated with both coeliac disease and dilated cardiomyopathies may explain the lack of association reported in others studies, mostly based on small sample size.

Bardella et al. [14], assessed the prevalence of cardiac involvement in patients with inflammatory bowel disease and in patients with celiac disease: the prevalence was similar, indicating that cardiac damage is not specific for celiac disease.

Other authors show that anti-transglutaminase antibodies can be found in cardiological patients, and these antibodies are related to the severity or extension of the myocardial tissue lesion, suggesting a loss of specificity for these antibodies in celiac disease [15]; in rat models of cardiac failure in fact an upregulation of mRNA for antitissue transglutaminase has been reported [16].

\section{Returning to our patient}

Dr. Boldrini: Going back to our patient and his early cardiac 1 history, in 1998 a coronary angiography was performed, but it did not reveal any vascular alteration. Laboratory tests were performed in 1997 and showed hypoalbuminemia ( $3 \mathrm{~g} / \mathrm{dl})$ and iron deficiency: no further etiologic investigations were performed on that occasion. We can only hypothesize that the patient might have presented at that time early signs of an incipient celiac disease.

This hypothesis could be supported by literature data showing that only $25 \%$ of celiac patients have a history of typical gastrointestinal symptoms, while iron deficiency is the most common manifestation of the disease and is present in $75 \%$ of cases [14].

Ventura et al. [17] examined the relationship between the prevalence of autoimmune disorders in celiac disease and the duration of exposure to gluten: the prevalence of autoimmune disorders in celiac disease is related to the duration of gluten exposure.

Possibly, cardiomyopathy in our patient was the result of very long gluten introduction and appeared as an autoimmune-related response to celiac disease. We may also wonder whether a link exists between the advanced cardiac disease (represented by dyssynchrony) and the current "major symptoms" of celiac disease [18].

Six weeks after good adherence to a gluten-free diet, the patient presented with persistent abdominal pain and diarrhoea; abdominal TC showed mesenteric thickening and enlarged mesenteric lymph nodes the histopathologic examination of jejunal and duodenal lesions were negative for lymphoma.

\section{Conclusion}

Prof. Pietrangelo: In summary, considering the available literature data, a possible association between celiac disease and cardiomyopathy clearly emerges. Unfortunately in our patient, neither endomyocardial biopsy nor cardiac MRI was performed to clearly prove cardiac involvement and reach a definite diagnosis.

In conclusion, the idiopathic dilated cardiomyopathy of our patient could represent the end stage of an inflammatory autoimmune cardiomyopathy; if so, the gluten-free diet could represent a tool to stop the autoimmune antimyocardial phenomena leading to subsequent improvement of clinical and ventricular functionality. This case clearly indicates that it is advisable to offer to all patients with cardiomyopathy and celiac disease an endomyocardial biopsy for a better histological and diagnostic definition.

What might be the role of immunosuppressor therapy associated with gluten-free diet? This case report notes the importance of the pathogenetic link between celiac disease and cardiomyopathy and warrants future studies on intestinal and myocardial autoimmunity.

Conflict of interest None. 


\section{References}

1. Curione M, Barbato M, De Biase L et al (1999) Prevalence of coeliac disease in idiopathic dilated cardiomyopathy. Lancet 354(9174):222-223

2. De Bem RS, Da Ro Sa Utiyama SR, Nisihara RM et al (2006) Celiac disease prevalence in Brazilian dilated cardiomyopathy patients. Dig Dis Sci May 51(5):1016-1019

3. Frustaci A, Cuoco L, Chimenti C et al (2002) Celiac disease associated with autoimmune myocarditis. Circulation 105(22): 2611-2618

4. Prati D, Bardella MT, Peracchi M et al (2002) North Italy Transplant Programme Working Group (NITp). High frequency of anti-endomysial reactivity in candidates to heart transplant. Dig Liver Dis 34(1):39-43

5. Chimenti C, Pieroni M, Frustaci A (2001) Celiac disease in idiopathic dilated cardiomyopathy. Ital Heart J 2:658-659

6. Goel NK, McBane RD, Kamath PS (2005) Cardiomyopathy associated with celiac disease. Mayo Clin Proc 80(5):674-676

7. van Elburg RM, Uil JJ, Mulder CJ, Heymans HS (1993) Intestinal permeability in patients with coeliac disease and relatives of patients with coeliac disease. Gut 34:354-357

8. Hall EJ, Batt RM (1991) Abnormal permeability precedes the development of a gluten sensitive enteropathy in Irish setter dogs. Gut 32:749-753

9. Chimenti C, Pieroni M, Maseri A, Frustaci A (2002) Dilated cardiomyopathy and celiac disease. Ital Heart J 3:385

10. Not T, Faleschini E, Tommasini A et al (2003) Celiac disease in patients with sporadic and inherited cardiomyopathies and in their relatives. Eur Heart J 24(15):1455-1461
11. Curione M, Danese C, Viola F, Di Bona S, Anastasia A, Cugini P, Barbato M (2005) Carnitine deficiency in patients with coeliac disease and idiopathic dilated cardiomyopathy. Nutr Metab Cardiovasc Dis 15(4):279-283

12. Elfström P, Hamsten A, Montgomery SM, Ekbom A, Ludvigsson JF (2007) Cardiomyopathy, pericarditis and myocarditis in a population-based cohort of inpatients with coeliac disease. J Intern Med 262(5):545-554

13. Vizzardi E, Lanzarotto F, Carabellese $N$ et al (2008) Lack of association of coeliac disease with idiopathic and ischaemic dilated cardiomyopathies. Scand J Clin Lab Invest 68(8):692-695

14. Bardella MT, Cantù P, Cesana BM, Piodi LP (2004) Severe heart disease in coeliac sprue and inflammatory bowel disease: a common pathogenesis? Scand J Gastroenterol 39(12):1304-1305

15. Di Tola M, Barillà F, Trappolini M et al (2008) Antitissue transglutaminase antibodies in acute coronary syndrome: an alert signal of myocardial tissue lesion? J Intern Med 263(1):43-51

16. Iwai N, Shimoike H, Kinoshita M (1995) Genes up-regulated in hypertrophied ventricle. Biochem Biophys Res Commun 209:527-534

17. Ventura A, Magazzù G, Greco L (1999) Duration of exposure to gluten and risk for autoimmune disorders in patients with celiac disease. SIGEP Study Group for Autoimmune Disorders in Celiac Disease. Gastroenterology 117(2):297-303

18. Di Sabatino A, Corazza GR (2009) Coeliac disease. Lancet 373(9673):1480-1493 